\title{
Article \\ The Development of SiPM-Based Fast Time-of-Flight Detector for the AMS-100 Experiment in Space
}

\author{
Chanhoon Chung * (D), Theresa Backes, Clemens Dittmar, Waclaw Karpinski, Thomas Kirn, Daniel Louis, \\ Georg Schwering, Michael Wlochal and Stefan Schael
}

check for updates

Citation: Chung, C.; Backes, T.; Dittmar, C.; Karpinski, W.; Kirn, T.;

Louis, D.; Schwering, G.; Wlochal, M.;

Schael, S. The Development of

SiPM-Based Fast Time-of-Flight

Detector for the AMS-100 Experiment

in Space. Instruments 2022, 6, 14.

https://doi.org/10.3390/

instruments6010014

Academic Editors: Matteo Centis-Vignali, Eraldo Oliveri,

Christopher Betancourt and Antonio Ereditato

Received: 15 November 2021

Accepted: 10 February 2022

Published: 13 February 2022

Publisher's Note: MDPI stays neutral with regard to jurisdictional claims in published maps and institutional affiliations.

Copyright: (C) 2022 by the authors. Licensee MDPI, Basel, Switzerland. This article is an open access article distributed under the terms and conditions of the Creative Commons Attribution (CC BY) license (https:// creativecommons.org/licenses/by/ $4.0 /)$
Physics Institute, RWTH Aachen University, D52056 Aachen, Germany; theresa.backes@rwth-aachen.de (T.B.); clemens.dittmar@rwth-aachen.de (C.D.); karpinski@physik.rwth-aachen.de (W.K.); kirn@physik.rwth-aachen.de (T.K.); louis@physik.rwth-aachen.de (D.L.); schwerin@physik.rwth-aachen.de (G.S.); wlochal@physik.rwth-aachen.de (M.W.); schael@physik.rwth-aachen.de (S.S.)

* Correspondence: chchung@physik.rwth-aachen.de; Tel.: +49-241-8027373

\begin{abstract}
AMS-100 is the next-generation high-energy cosmic-ray experiment in Space. It is designed as a magnetic spectrometer with a geometrical acceptance of $100 \mathrm{~m}^{2} \cdot \mathrm{sr}$ to be operated for ten years at the Sun-Earth Lagrange Point 2. Its Time-of-Flight (TOF) detector is a crucial sub-detector for the main trigger and the particle identification constructed from individual scintillation counters. A fast time measurement with a resolution of 20 ps for a single counter is required to cover wide energy ranges for particle identification. A prototype counter has been designed based on a fast plastic scintillator tile readout by two silicon photomultipliers (SiPMs). An amplifier board was built to merge $16 \mathrm{SiPM}$ channels into four readout channels in a hybrid connection. The signals are read out by a fast waveform digitizer. The timing performance was studied with electrons from a ${ }^{90} \mathrm{Sr}$ source. A time resolution of $40 \mathrm{ps}$ for a single counter has been achieved. Various operational and environmental conditions have been studied.
\end{abstract}

Keywords: TOF; SiPM; fast scintillator; AMS-100

\section{Introduction}

AMS-100 [1] is the next-generation high-energy cosmic-ray experiment that is planned to succeed the currently operating AMS-02 [2] aboard the International Space Station. It serves as a cosmic-ray observatory deployed near the Sun-Earth Lagrange Point 2 for the next decade.

As illustrated in Figure 1a, AMS-100 consists of a stack of cylindrical sub-detectors with a radius of $2 \mathrm{~m}$ and a length of $6 \mathrm{~m}$, surrounded by a magnet and a compensation coil, and it is equipped with external solar panels, a radiator, sun-shield and a space craft service module.

Along the instrument, time-of-flight (TOF) detectors reside on the inside and outside of the solenoid magnet, as illustrated in Figure $1 \mathrm{~b}$.

The TOF covers a cylindrical surface with a length of $6 \mathrm{~m}$ and a radius of $2 \mathrm{~m}$ resulting in a total area of $75 \mathrm{~m}^{2}$. It consists of four separate layers. Each layer is constructed from individual scintillator tiles coupled to two SiPMs with a time resolution of $\sigma_{t}=20 \mathrm{ps}$, where $\sigma_{t}$ is defined in Equation (2). The time resolution of the TOF system is $\sigma_{t} / 2$. This time resolution allows identifying cosmic ray isotopes, such as ${ }^{2} \mathrm{H},{ }^{3} \mathrm{He},{ }^{10} \mathrm{Be}$, with high accuracy over a wide range of momenta.

Recently, the PANDA [3] and MEG II [4] experiments have developed timing detectors based on a scintillator tile readout by a series of SiPMs and obtained a time resolution in the range of $\sigma_{t}=50 \sim 60 \mathrm{ps}$ [3-5]. In this article, we have adopted similar design concepts targeting a better time resolution mainly due to the enlarged active area of the SiPMs. In addition, we expect reduced electronic noise under the cold environmental temperature of $200 \mathrm{~K}$, at which the TOF will be operated in AMS-100. 


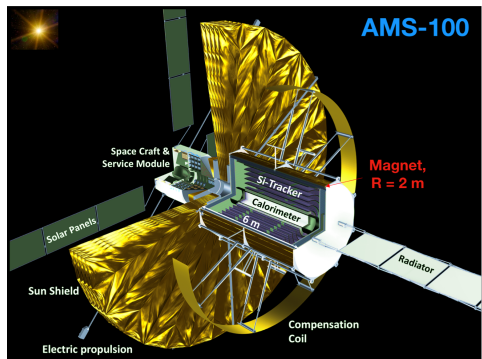

(a)

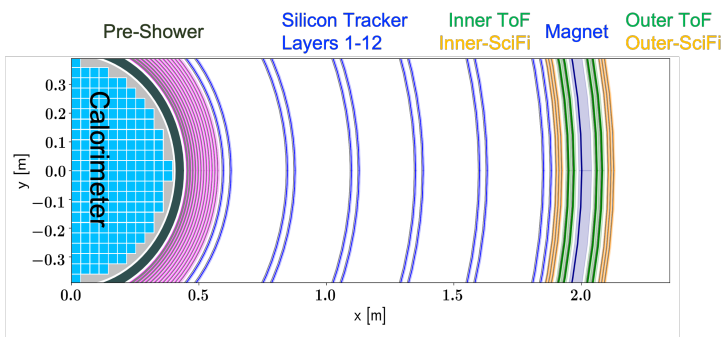

(b)

Figure 1. (a) A rendering of AMS-100 deployed in Space: The AMS-100 instrument has a cylindrical geometry with a radius of $2 \mathrm{~m}$ and a length of $6 \mathrm{~m}$, surrounded by a compensation coil. In front of the sun-shield, it is equipped with solar panels, electrical propulsion system and a space craft service module. The rear radiator removes the heat from the instrument. (b) Cross-section of the AMS-100 detector with its cylindrical sub-detector layers.

\section{SiPM-Based Time-of-Flight (Tof) Detector}

Similar to the PANDA and the MEG II experiments, a plastic scintillator tile with a short scintillation decay time and high photon statistics for fast timing measurements has been chosen.

The major contribution to the time resolution $\left(\sigma_{t}^{p h}\right)$ from photon statistics, as detected by the SiPM, can be expressed as [6]

$$
\sigma_{t}^{p h} \propto \sqrt{\frac{\tau_{r} \tau_{d}}{N_{d e t}}} \propto \sqrt{\frac{\tau_{r} \tau_{d}}{N_{p h} \cdot Q E \cdot C E \cdot G}}
$$

where $\tau_{r}$ and $\tau_{d}$ are the scintillator's rise and decay time, and $N_{d e t}$ is the number of detected photons. $N_{\text {det }}$ can be expressed as a product of the number of photons $\left(N_{p h}\right)$, the quantum efficiency $(\mathrm{QE})$, the collection efficiency $(\mathrm{CE})$ and the gain $(\mathrm{G})$ of SiPM. The product of $\mathrm{QE} \cdot \mathrm{CE}$ is widely defined as the photon-detection efficiency (PDE). Here, noise contributions from crosstalk, afterpulse, dark current and electronic noise are excluded. According to Equation (1), the time resolution improves as $\tau_{r}$ and $\tau_{d}$ becomes shorter.

For fast timing purposes, polyvinyltoluene-based Eljen-228 and Eljen-232 plastic scintillators are selected because of their high light yield of 10,200 photons $/ 1 \mathrm{MeV} \mathrm{e}^{-}$, low density of $1.023 \mathrm{~g} / \mathrm{cm}^{3}$ and fast scintillation rise and decay times of less than $500 \mathrm{ps}$ and $1600 \mathrm{ps}$, respectively. The given refractive index is 1.58 , and the light output is constant in the temperature range of $-60{ }^{\circ} \mathrm{C}$ to $+20^{\circ} \mathrm{C}$ [7]. The dimensions of the used scintillator tile are $25 \mathrm{~mm}$ (width) $\times 6 \mathrm{~mm}$ (thickness) $\times 120 \mathrm{~mm}$ (length). The thickness is adjusted to match the height of the single-channel SiPM.

A SiPM is a semiconductor-based photon detector that consists of a rectangular array of microcells. Each microcell consists of an avalanche photodiode (APD) operating in Geiger mode and a quenching resistor. All of the APDs and quenching resistors are identical, and microcells are connected to one common output in parallel. Nowadays, the SiPM is a well-known photo sensor as its technology has reached impressive maturity. Similar to the conventional photomultiplier tube, the SiPM provides a fast response. Excellent properties such as compactness, high gain, low cost and high immunity to magnetic fields enable a flexible and highly segmented design for the TOF detector layout in large-scale experiments [8]. Low bias voltage and low power consumption are additional advantages for a long-term operation in Space.

For fast SiPMs, the S14161 series from Hamamatsu Photonics (HPK) is chosen. Compared to the S13360 series used by PANDA [3], it provides a higher PDE ( $50 \%$ at $\lambda_{\text {peak }}=450 \mathrm{~nm}$, $V_{\text {bias }}=V_{B D}+2.7 \mathrm{~V}$ ) with low crosstalk, a higher gain in the order of $10^{6}$ and a lower breakdown voltage $\left(V_{B D}=38 \mathrm{~V}\right)$. The afterpulse effects and dark counting rate are also significantly reduced by two orders of magnitude compared to the S12571/S12572 series [9]. 
This SiPM consists of $N=16(4 \times 4)$ channels. Each channel has an effective area of $(6 \times 6) \mathrm{mm}^{2}$ with a pixel pitch of $50 \mu \mathrm{m}$, leading to a large dynamic range due to the high number of microcells (14 k).

There are several ways to combine the N SiPM channels, such as series, parallel and hybrid connections, as described in detail in reference [3]. The optimal scenario, which is used in this study, is the hybrid scheme. It makes use of the parallel connection feature of a common $V_{\text {bias }}$ among the $N$ channels and the series connection feature relevant to the signal quality but with the use of a decoupling capacitor between the channels.

\section{Experimental Setup}

A TOF counter prototype has been built to quantify the time resolution. As illustrated in Figure 2a, a single scintillator tile is read out by two SiPM arrays mounted on two opposite edges of a $120 \mathrm{~mm}$ long scintillator. Each SiPM consists of four channels in a row, as depicted in Figure $2 b$.

Compared with the PANDA TOF detector, the enlarged active area of the SiPM is expected to improve the time resolution by a factor $\simeq 2$ given by the different surface coverage [10], which improves the number of detected photons $\left(N_{d e t}\right)$ accordingly (Equation (1)).

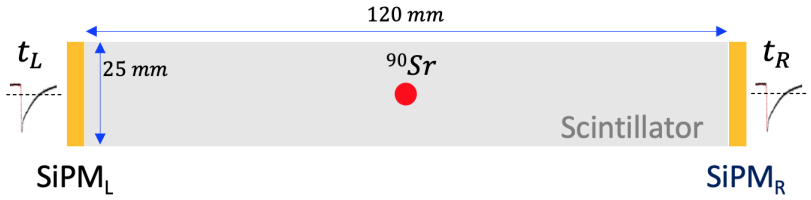

(a)

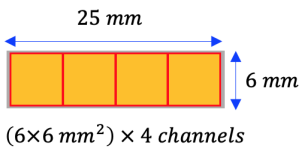

(b)

Figure 2. A TOF test counter consists of a scintillator and two SiPMs coupled to the scintillator at opposite sides. (a) Top view: A radioactive source, ${ }^{90} \mathrm{Sr}$, is placed at the middle of scintillator. The scintillation light is collected at both SiPMs. (b) Side view: Each side of the scintillator is fully faced with four SiPM channels.

In this article, we use both the coincidence time resolution $\left(\sigma_{\Delta t}\right)$ and the time resolution $\left(\sigma_{t}\right)$, which are defined as

$$
\begin{aligned}
\Delta t & =\left(t_{L}-t_{R}\right), \quad \sigma_{\Delta t}=\sqrt{\sigma_{t_{L}}^{2}+\sigma_{t_{R}}^{2}} \\
t=\frac{\left(t_{L}+t_{R}\right)}{2}, & \sigma_{t}=\frac{1}{2} \cdot \sqrt{\sigma_{t_{L}}^{2}+\sigma_{t_{R}}^{2}}=\frac{1}{2} \cdot \sigma_{\Delta t}
\end{aligned}
$$

where $t_{L, R}$ denotes the time stamp of the left or right SiPM, and $t$ is the average time for a coincident event, as shown in Figure 2a.

\subsection{Design of DAQ}

A schematic of the experimental test setup is depicted in Figure 3. It shows a TOF prototype consisting of a scintillator tile coupled to SiPMs and front-end boards inside a climate chamber. The interior of the climate chamber is light-tight to avoid any background light on the scintillator. Signal, power, communication and other service lines are interconnected with external instruments through a dedicated feedthrough.

The signals induced by an LED or a radioactive source are fed into the front-end board and then read out by a high-speed Domino Ring Sampling (DRS4) waveform digitizer [11]. The DRS4 evaluation board is capable of digitizing four input channels simultaneously at sampling rates of up to 5 Giga-samples per second with 1024 sampling points per channel. This is sufficient to resolve picosecond (ps) timing. Advanced internal time calibration circuits allow obtaining the sub-ps time resolution. The board is powered through the USB port and contains an onboard trigger logic module. 


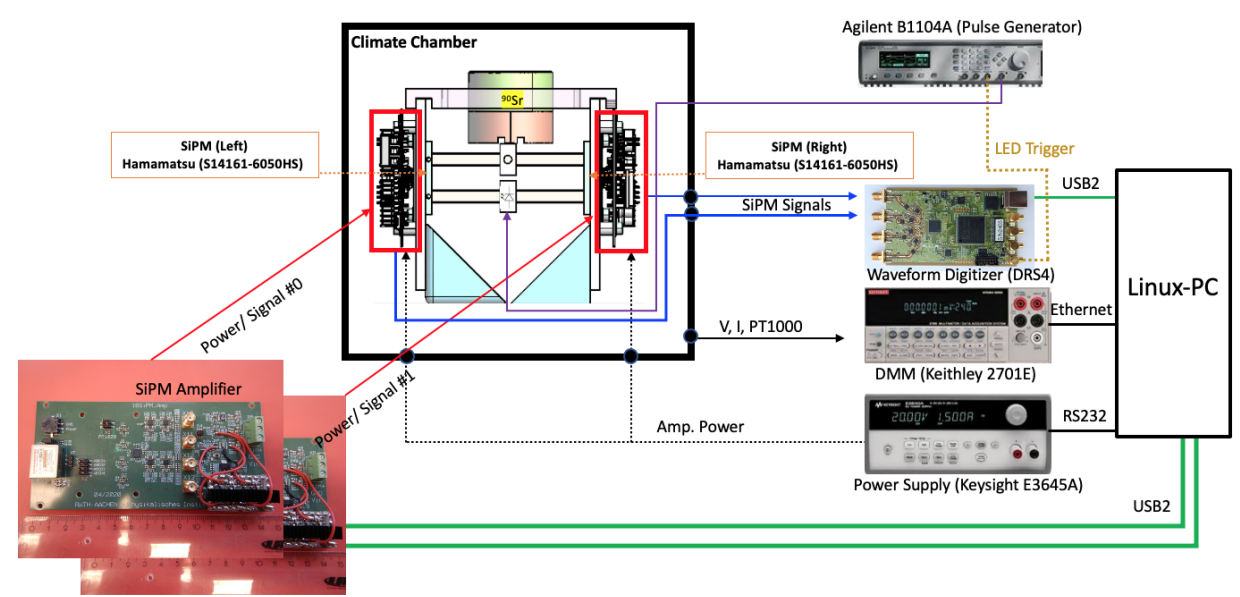

Figure 3. TOF prototype test setup inside a climate chamber. An LED driven by a pulse generator is used as a light source, and a radioactive source is used to provide signals equivalent to MIPs. The SiPM output signals are amplified and then fed into a high-speed waveform digitizer (DRS4). All devices are controlled by a linux-based PC.

A preamplifier board is used to control the gain of two phases of Op-amp. An onboard power supply module (Hamamatsu C11204-01 [12]) provides the bias voltage for the SiPM. A USB micro-controller (FTDI-FT4222H [13]) is used to read out a temperature sensor on the SiPM. The bias power supply module has a built-in temperature compensation function so that the SiPM can be operated in optimum conditions even in environments of varying temperatures. It is controlled via a USB FT4222H bridge with a serial peripheral interface (SPI) protocol.

All monitoring data of voltages, external power supply current, temperatures and other operating conditions of the climate chamber are recorded by a Digital Multi-Meter (DMM, Keithley 2700E) interfaced to a LabVIEW program. A linux-based PC is used to control and monitor all hardware devices.

\subsection{Ultraviolet (UV) LED and ${ }^{90} \mathrm{Sr}$ Radioactive Source}

Figure 4 shows the prototype test setup inside the climate chamber. An ultraviolet (UV) light LED diode ( $375 \mathrm{~nm}$, Thorlabs LED375L) is placed in the middle of the scintillator, as shown in Figure 4a. It is used to generate ultra-short light pulses of low power $(<1 \mathrm{~mW})$ and $10 \mathrm{~ns}$ width (FWHM) within a narrow viewing angle $\left( \pm 20^{\circ}\right)$. The light is transmitted by total reflection towards the SiPMs. The LED is mounted on a mechanical holder with a pinhole ( $2 \mathrm{~mm}$ diameter) that serves as a collimator of the produced light pulses. The UV-LED has been used to quantify the contribution of the readout electronics to the time resolution. We obtained a contribution of about $5 \mathrm{ps}$, which is, at present, negligible compared to the observed total time resolution of $\simeq 40$ ps described in Section 4.6.

To evaluate the performance of the prototype, a strontium $\left({ }^{90} \mathrm{Sr}\right)$ radioactive source is used, as shown in Figure $4 \mathrm{~b}$. The electrons emitted by its daughter, yttrium $\left({ }^{90} \mathrm{Y}\right)$, have a continuous energy distribution with a maximum energy of $2.28 \mathrm{MeV}$. The electrons are collimated with a pin-hole ( $2 \mathrm{~mm}$ diameter) at the bottom of the aluminum housing container. The electrons deposit energy in the scintillator and the resulting light is detected by both SiPMs. The SiPMs are read out by the DRS4. The threshold of its discriminator is adjusted at a low level of $\sigma_{\text {noise }} \times 20 \approx 10 \mathrm{mV}$. The recorded data are used for offline analysis. In rare cases, the electron can release its maximum energy of $2.28 \mathrm{MeV}$ in the scintillator. The contribution is estimated using the maximum of the signal pulse spectrum in a self-triggered mode to be less than $1 \%$.

Electrons in the $1 \sim 3 \mathrm{MeV}$ energy range deposit $2 \mathrm{MeV} \mathrm{cm}^{2} / \mathrm{g}$ in a vinyltoluene-based plastic scintillator [14] equivalent to an MIP. Therefore, in this report, we assume that an electron of $1 \mathrm{MeV}$ energy deposits the same energy as an MIP. 


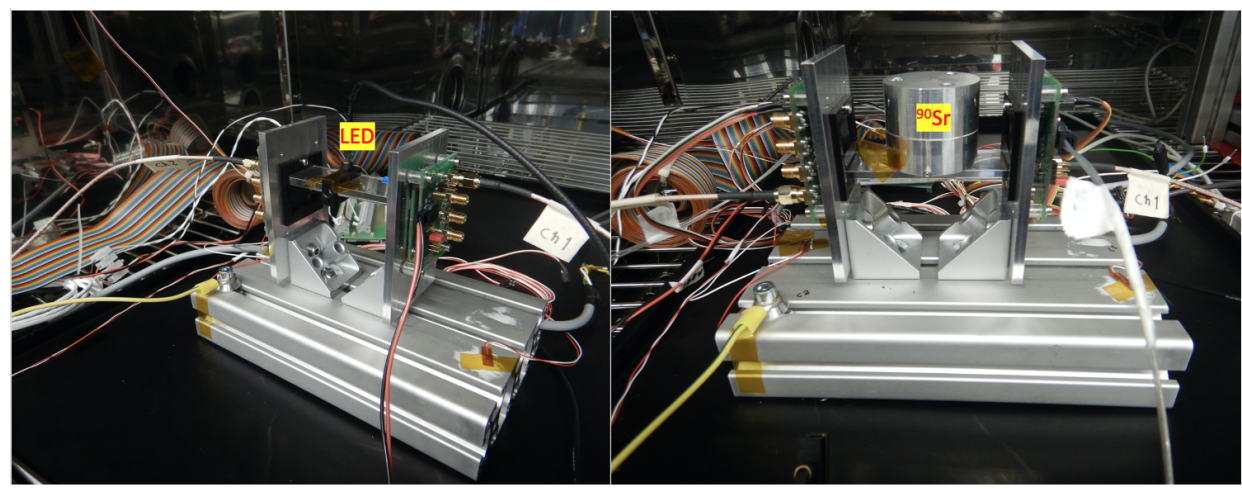

(a)

(b)

Figure 4. The TOF prototype test setup: (a) A LED $(375 \mathrm{~nm})$ injects the light into the scintillator for systematic study; (b) on top of the scintillator surface, a radioactive source $\left({ }^{90} \mathrm{Sr}\right)$ is employed to investigate the performance of the prototype.

\subsection{Breakdown Voltage and Temperature Dependency}

The breakdown voltage $\left(V_{B D}\right)$ decreases at low temperatures due to the larger carrier mobility. It can be determined from the measurements of the pulse height as a function of the bias voltage $\left(V_{\text {bias }}\right)$. It is derived as the intercept on the $V_{\text {bias }}$ axis. The $V_{B D}$ has been determined as $38.1 \mathrm{~V}$ for the left SiPM and $37.8 \mathrm{~V}$ for the right SiPM at $+23{ }^{\circ} \mathrm{C}$.

In a wide range of environmental temperatures, the $V_{B D}$ is linearly correlated with the temperature (see Figure 5):

$$
V_{B D}(T)=V_{B D}\left(T_{0}\right)+\alpha \cdot\left(T-T_{0}\right)
$$

where $\alpha$ denotes the temperature coefficient, and $V_{B D}\left(T_{0}\right)$ is the breakdown voltage at the reference temperature $T_{0}$. The measured average temperature coefficient is $\alpha=$ $(34.2 \pm 0.1 \pm 0.3) \mathrm{mV} /{ }^{\circ} \mathrm{C}$. The errors represent the statistical and systematic uncertainty, respectively. A systematic error of $1 \%$ was estimated by varying the fit ranges to determine a breakdown voltage at a given temperature. It is consistent with the reference value of $34 \mathrm{mV} /{ }^{\circ} \mathrm{C}$ provided by the vendor [9].

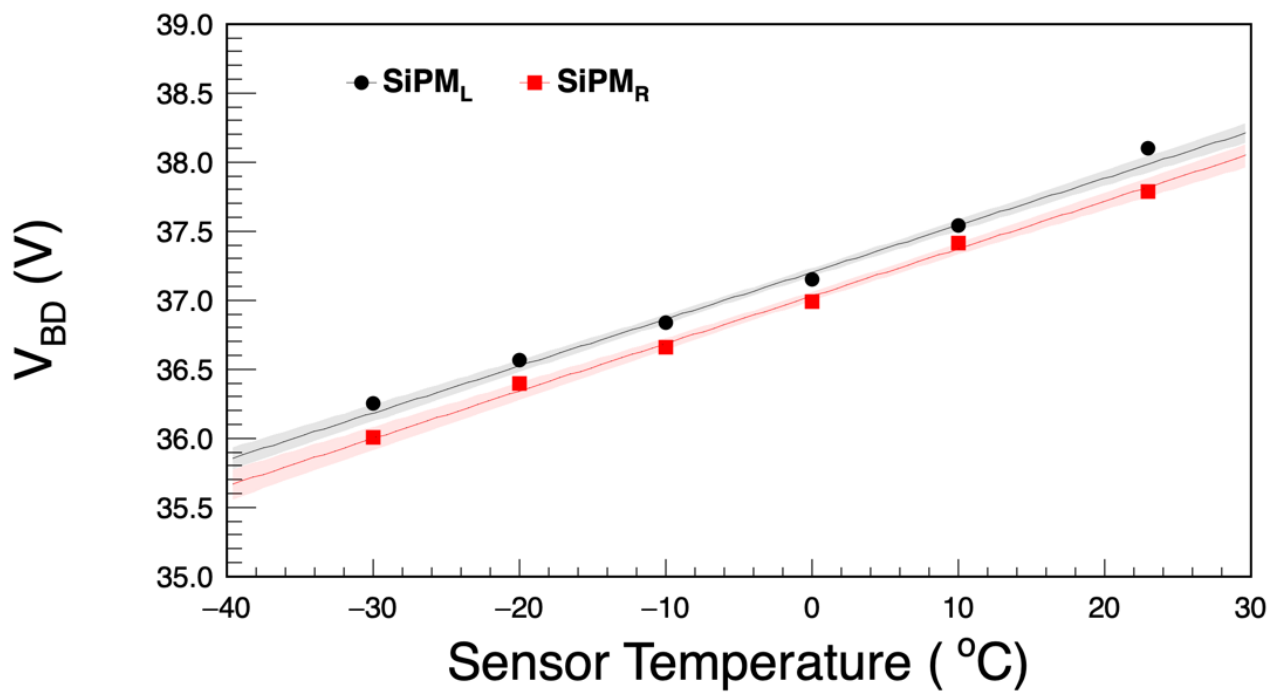

Figure 5. Dependence of the breakdown voltage $\left(V_{B D}\right)$ on temperature for two SiPMs. The breakdown voltage is obtained by measuring signal pulse heights with a ${ }^{90} \mathrm{Sr}$ source. As the temperature decreases from $+23{ }^{\circ} \mathrm{C}$ to $-30{ }^{\circ} \mathrm{C}$, the $V_{B D}$ decreases from $38 \mathrm{~V}$ to $36 \mathrm{~V}$. The black and red error bands indicate the $95 \%$ confidence level of the fits. 
The power supply module that is implemented with a temperature-voltage feedback loop generates the bias voltage according to the following formula.

$$
V_{\text {bias }}(T)=V_{\text {bias }}\left(T_{0}\right)+\alpha \cdot\left(T-T_{0}\right)
$$

The total charge of the signal is proportional to the gain. The gain $(G)$ increases linearly with the overvoltage and is determined by

$$
G=C_{c e l l} \cdot \Delta V=C_{c e l l} \cdot\left(V_{b i a s}-V_{B D}\right)
$$

where $C_{c e l l}$ denotes the SiPM microcell capacitance. If we assume that the temperature gradient of $C_{c e l l}$ is negligible, the gain decreases with increasing temperature at the same bias voltage unless temperature compensation is applied.

\section{Data Analysis and Results}

\subsection{Fast Leading Edge Signals}

Fast leading edges of the signals are fitted to estimate the time constant, rise time, baseline and height of the pulse after baseline subtraction. Figure 6 illustrates the raw signals at $\Delta V=3 \mathrm{~V}$ induced by the ${ }^{90} \mathrm{Sr}$ source and recorded by both SiPMs. Each signal shape is well described by a step function of the form

$$
f(t)=a+\frac{b}{\left(1+e^{-\left(t-t_{0}\right) / t_{\tau}}\right)}
$$

where $a$ and $b$ describe the pulse baseline and the amplitude of the signal, respectively. $t_{0}$ refers to the time at half maximum of pulse height and $t_{\tau}$ to a time constant. The rise time is estimated by the time needed for the pulse amplitude to rise from $10 \%$ to $90 \%$ of its maximum.

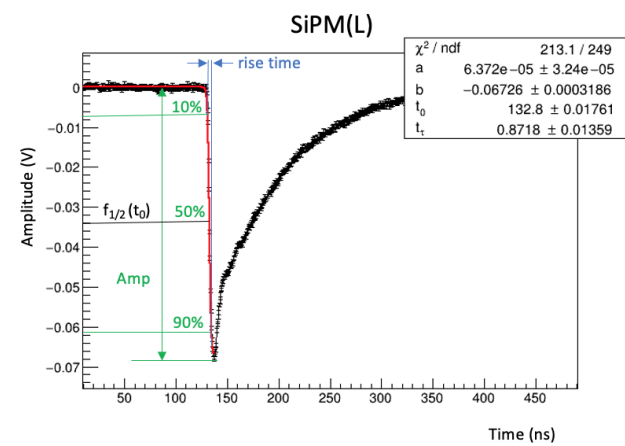

(a)

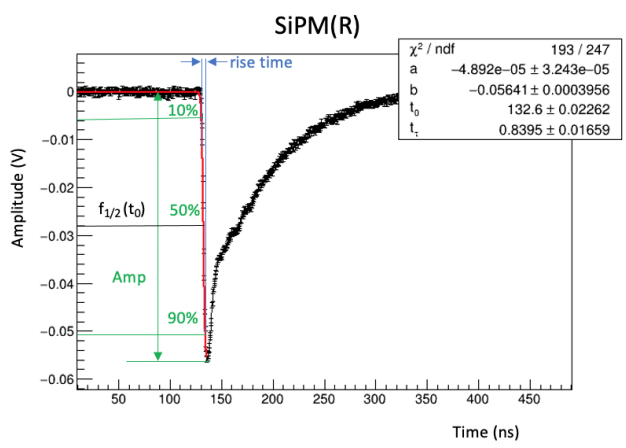

(b)

Figure 6. Fast pulse signals induced by the ${ }^{90} \mathrm{Sr}$ source and recorded by both SiPMs: (a) left SiPM(L) and (b) right $\operatorname{SiPM}(\mathrm{R})$. The fit result (red lines) on the fast leading edge of the signals, the rise time and the time at half maximum of the pulse height, $t_{0}$, are also shown.

Similarly, the decay time is defined as the time needed for the pulse amplitude to decrease from $90 \%$ to $10 \%$ of its maximum. The total collected charge is estimated from the area between the graph and the time axis.

\subsection{Coincidence Time Resolution (CTR)}

The accuracy of the timing measurements is limited by jitter due to noise and timewalk from amplitude variations. The contribution of jitter to the time resolution is generally expressed to first approximation as

$$
\sigma_{t}^{j i t t e r}=\frac{\sigma_{n}}{d V / d t} \approx \frac{\sigma_{n}}{V_{\text {peak }} / t_{r}}=\frac{t_{r}}{S N R}
$$


where $\sigma_{n}$ denotes the electronic noise, $d V / d t$ is the slope of the signal amplitude at a given threshold, which can be approximated as the ratio of peak amplitude $\left(V_{\text {peak }}\right)$ to rise time $\left(t_{r}\right)$, and $S N R$ is the signal-to-noise ratio. Consequently, the time resolution improves directly with decreasing signal rise time and increasing peak signal amplitude.

The best time resolution is obtained from a constant fractional timing method as it eliminates an amplitude-dependent time-walk for signals $[15,16]$. In this article, this method is implemented as a constant fractional pulse height trigger routine in the offline waveform analysis.

In Figure 7, the distributions of the time constant, the rise time and the time stamps for both left and right SiPMs are presented at a constant fractional threshold of $0.2(20 \%)$. The raw data are collected with a $70 \mathrm{mV}$ trigger out of a maximum pulse height of $170 \mathrm{mV}$, which is estimated at the maximum electron energy of $2.28 \mathrm{MeV}$. Events are selected for the coincident pairs of signals within a time difference window fixed at $10 \mathrm{~ns}$ with an efficiency of $99.9 \%$. The measured timing distributions are fitted using an asymmetric Gaussian function with mean value $\mu$, amplitude $C$ and its standard deviation $\sigma$ defined as

$$
f(t)=C \cdot \exp \left(-\frac{(t-\mu)^{2}}{\sigma^{2}}\right)
$$

where $\sigma=\sigma_{1}$ if $t<\mu$ and $\sigma=\sigma_{2}$ otherwise.

The time constant and the rise time are identical for both SiPMs and have a value of $0.8 \mathrm{~ns}$ and $3.6 \mathrm{~ns}$, respectively.
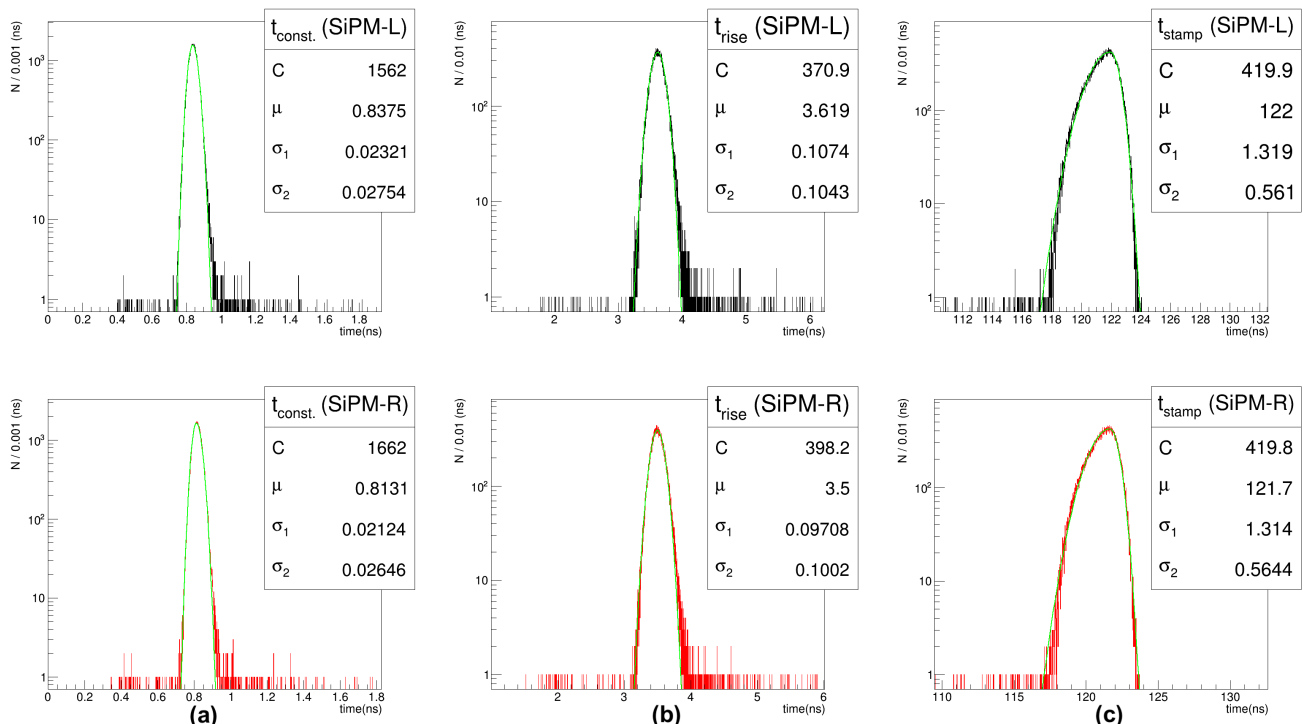

Figure 7. Measured timing information from both SiPMs for the Eljen-228 scintillator. Each distribution shows the time constant (a), the rise time (b) and the time stamp (c) tagged at a given constant fractional threshold of $0.2(20 \%)$. The data are fitted with an asymmetric Gaussian function defined in Equation (8).

The time difference between coincident pairs of pulses, that is, between left and right SiPMs $\left(\Delta t=t_{\text {left }}-t_{\text {right }}\right)$, is shown in Figure 8 for two samples collected at low and high trigger levels of $20 \mathrm{mV}$ and $70 \mathrm{mV}$. Considering the core Gaussian fits, the measured CTR is $\sigma_{\Delta t}=(100 \pm 0.8)$ ps and $\sigma_{\Delta t}=(82 \pm 0.2)$ ps with an efficiency of $70 \%$ and $98 \%$, respectively. The systematic uncertainty is estimated to be $1 \%$ by repeating the measurement several times. It includes the effect of temperature variations of $\pm 0.1^{\circ} \mathrm{C}$ in the climate chamber and the source position of $\pm 2 \mathrm{~mm}$. The contribution of this systematic uncertainty on the fits is negligible. 

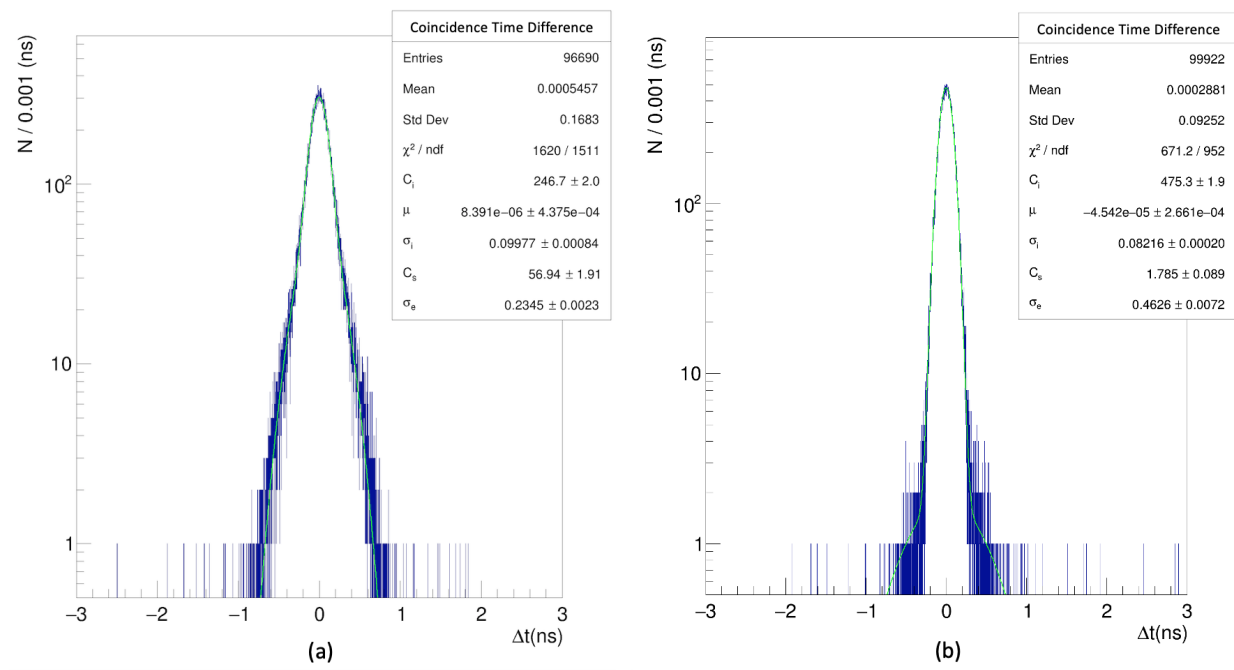

Figure 8. Distributions of time difference between left and right SiPMs $\left(\Delta t=t_{\text {left }}-t_{\text {right }}\right)$ for (a) $20 \mathrm{mV}$ low and (b) $70 \mathrm{mV}$ high thresholds for the Eljen-228 scintillator. Each distribution is fitted by the superimposition of two Gaussians with the same mean. The CTR represents the core standard deviation of the Gaussian function $\left(\sigma_{\Delta t}\right)$ and reveals $(100 \pm 0.8)$ ps and $(82 \pm 0.2)$ ps, respectively.

\subsection{Comparative Study of the CTR of Two Scintillators}

In Figure 9, we compare the CTR between Eljen-228 and Eljen-232 scintillators as a function of overvoltage. This study was performed by varying the overvoltage from $0.5 \mathrm{~V}$ up to $7 \mathrm{~V}$ at a fixed discriminator threshold of $10 \mathrm{mV}$ and a constant environmental temperature of $+23^{\circ} \mathrm{C}$. The minimum CTR is estimated to be equal to $\sigma_{\Delta t}=(96.4 \pm 0.8) \mathrm{ps}$ and $\sigma_{\Delta t}=(122.9 \pm 1.2)$ ps for Eljen-228 and Eljen-232, respectively. The results show a better performance for Eljen-228, which originates from a $20 \%$ higher light yield that overcompensates for the $30 \%$ longer rise time, compared to Eljen-232.

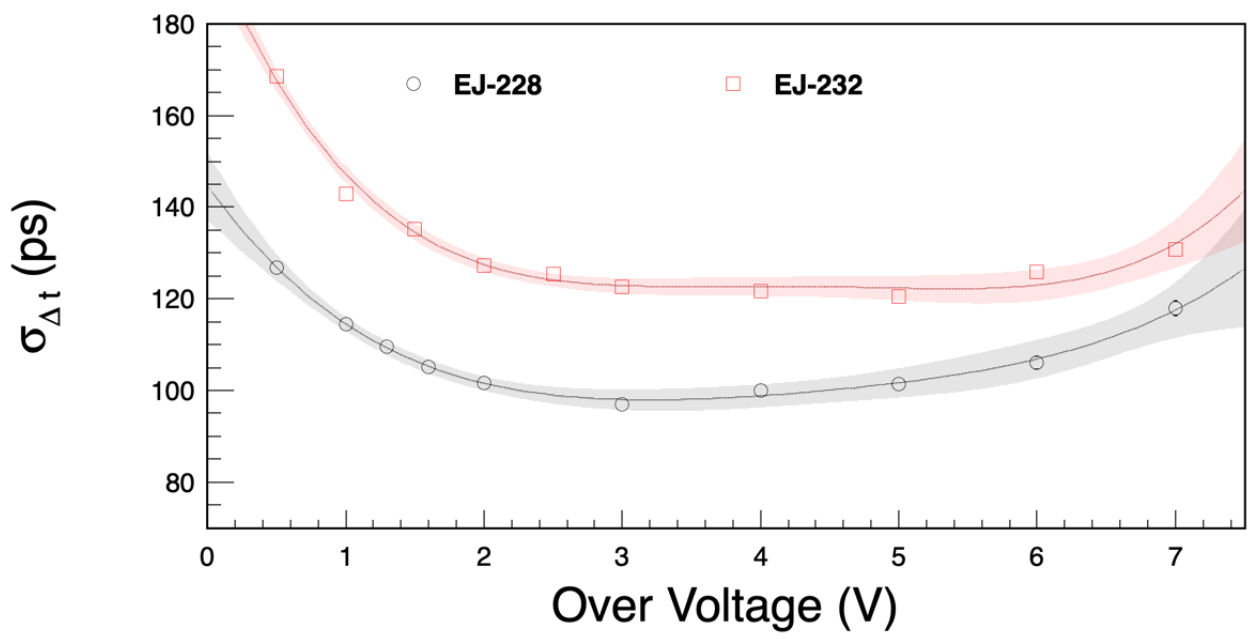

Figure 9. Comparison between Eljen-228 and Eljen-232. The overvoltage was varied from $0.5 \mathrm{~V}$ to $7 \mathrm{~V}$ at a fixed discriminator threshold of $10 \mathrm{mV}$ and an environmental temperature of $+23^{\circ} \mathrm{C}$. The gray and red error bands indicate the $95 \%$ confidence level of the fits.

A quantitive understanding of this result would require a detailed study with a MonteCarlo simulation to model the optical photon transport in the plastic scintillator coupled to the SiPM, as reported in the articles $[17,18]$.

The CTR is highly dependent on the bias voltage, trigger threshold level, optical couplings and temperature conditions. 
For the following CTR measurements, the scintillator (Eljen-228) is wrapped with a single layer of aluminized mylar to enhance scintillation light collection. The possible impact of optical coupling grease between the scintillator and the SiPMs was not investigated in this study.

\subsection{Position Dependency of Time Resolution}

The position dependency of the time resolution was studied in $5 \mathrm{~mm}$ steps in the longitudinal direction with a bias voltage of $41.0 \mathrm{~V}$ ( or $\Delta V=3.0 \mathrm{~V}$ ) and a discriminator threshold of $40 \mathrm{mV}$ to acquire electrons that are close to $\operatorname{MIPs}\left(E_{e^{-}} \simeq 1 \mathrm{MeV}\right)$. The time difference, $\Delta t$, of the two SiPMs can be expressed as a linear function of the source position as

$$
\begin{aligned}
& t_{L}\left(x_{i}\right)=t_{s}+\frac{\left(\frac{l}{2} \pm x_{i}\right)}{v_{e f f}}+t_{\text {trig.L }} \\
& t_{R}\left(x_{i}\right)=t_{s}+\frac{\left(\frac{l}{2} \mp x_{i}\right)}{v_{e f f}}+t_{\text {trig. } R} \\
& \Delta t\left(x_{i}\right)=t_{L}\left(x_{i}\right)-t_{R}\left(x_{i}\right)=\left(\frac{2}{v_{e f f}}\right) \cdot x_{i}+\Delta t_{\text {trig }}
\end{aligned}
$$

where $t_{s}$ represents the start time, $x_{i}$ is the position of the source with respect to the center of the scintillator and $l$ is the length of the scintillator. $t_{\text {trig. } L(R)}$ denotes the time stamp of each $\mathrm{SiPM}$, and $v_{\text {eff }}$ is the effective speed of light in the scinitllator.

As shown in Figure 10, we can derive the effective speed of light in the scintillator, $v_{\text {eff }}=19.1 \pm 0.1$ (stat) \pm 0.7 (syst) $\mathrm{cm} / \mathrm{ns}$, by fitting the data by a function linear in the source position. The systematic error is estimated by varying the constant fractional threshold by $0.1(10 \%)$. The measured $v_{\text {eff }}$ results in a refractive index of $n=c / v_{\text {eff }}=$ $1.57 \pm 0.01$ (stat) \pm 0.06 (syst), which is consistent with the value of $n=1.58$ given in the data sheet of the product.

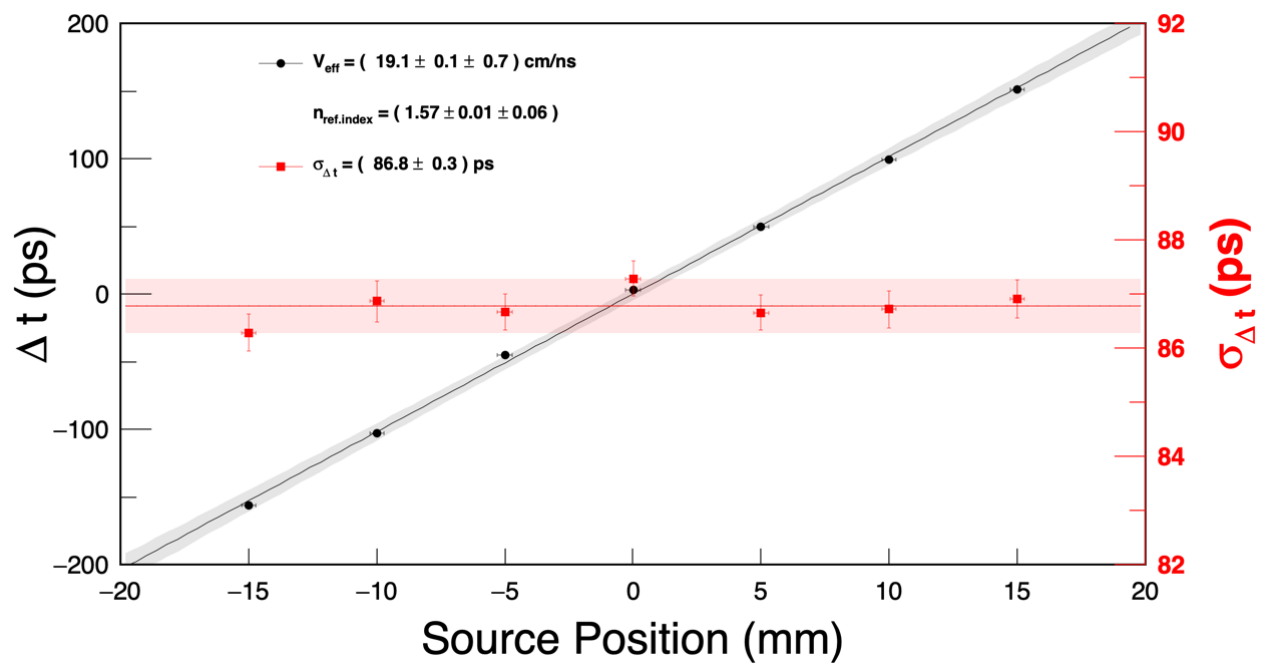

Figure 10. Time difference between left and right SiPMs as a function of source position on the scintillator. The CTR is calculated at a constant fractional threshold of $0.2(20 \%)$. The black and red error bands indicate the $95 \%$ confidence level of the fits.

The average CTR is equal to $(86.8 \pm 0.3)$ ps, which is equivalent to $\sigma_{t}=(43.4 \pm 0.1)$ ps. This indicates good uniformity along the longitudinal direction of the scintillator. This result can also be translated into a position resolution of $(8.29 \pm 0.02) \mathrm{mm}$. 


\subsection{Time Resolution vs. Bias Voltage}

At a low constant discriminator threshold of $10 \mathrm{mV}$, we varied the bias voltage $V_{\text {bias }}$ of the SiPM from $38.5 \mathrm{~V}$ to $45.0 \mathrm{~V}$ and recorded the pulse shapes at a temperature of $+23{ }^{\circ} \mathrm{C}$. This low threshold collects the events matching an average electron energy of $0.5 \mathrm{MeV}$. At each bias voltage, the fractional threshold level varies from 0.1 to 0.9 in a step of 0.1 . The obtained values for the CTR are shown in Figure 11.

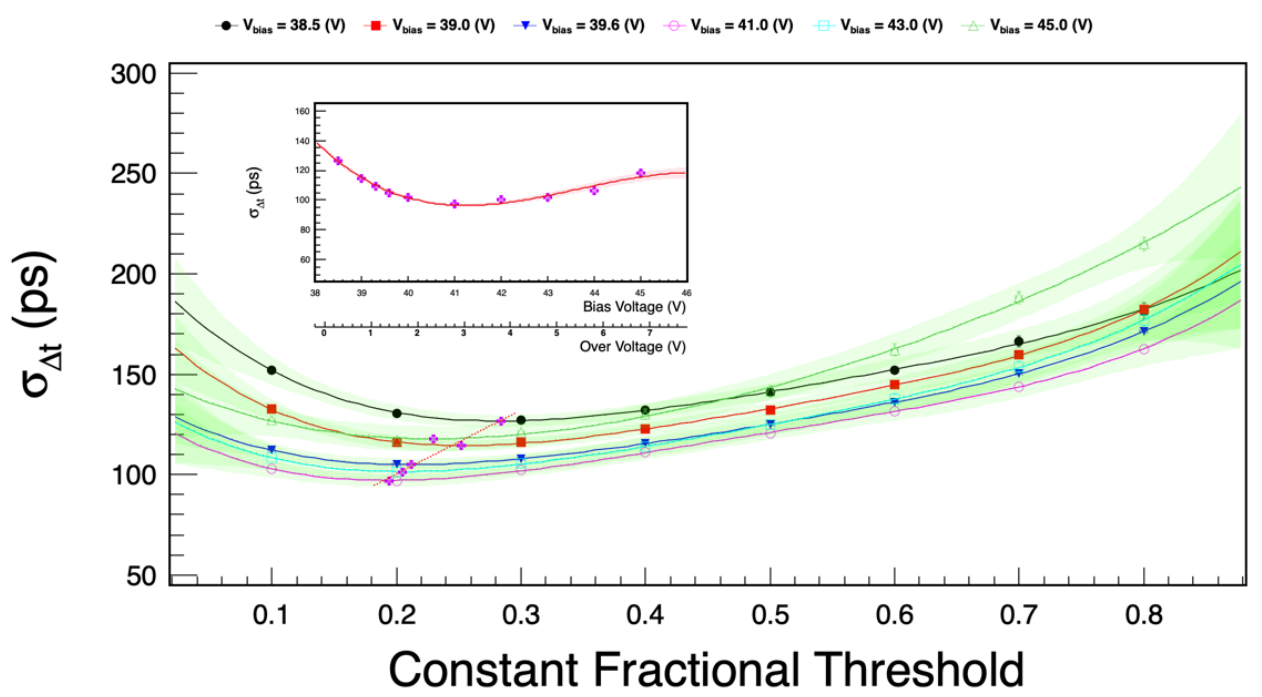

Figure 11. CTR as a function of the constant fractional threshold for different bias voltages. The minimum CTR values (magenta points) are distributed around a constant fractional threshold between $0.2(20 \%)$ and $0.3(30 \%)$. As depicted by a dotted linear fit line, it shifts from 0.3 to 0.2 as the bias voltage increases. The inlay indicates the minimum CTR as a function of the bias voltage and the overvoltage $(\Delta V)$. The best CTR for $0.5 \mathrm{MeV}$ electrons reveals $(96.4 \pm 0.4)$ ps at $\Delta V=3.25 \mathrm{~V}$. The colored error band indicates the $95 \%$ confidence level of the fits.

The minimum time resolution is obtained for a constant fractional threshold level between 0.2 and 0.3 anti-correlated with the bias voltage and consistent with the expectation from Equation (7) that the optimum time resolution is achieved when the pulse height derivative $(d V / d t)$ is at its maximum.

The time resolution manifestly improves when the bias voltage is increased to above $V_{B D}=38 \mathrm{~V}$ due to enhanced PDE, gain and SNR, as shown in Equations (1) and (7), but it starts deteriorating above $V_{\text {bias }}>43 \mathrm{~V}$ (or $\Delta V>5 \mathrm{~V}$ ) due to dark current noise, crosstalk and afterpulse effects, which are expected to increase with $\Delta V$ [19].

The best time resolution achieved is $\sigma_{t}=(48.2 \pm 0.2) p s$ at an overvoltage of $\Delta V=$ $3.25 \mathrm{~V}$.

\subsection{Time Resolution vs. Discriminator Threshold}

At a constant overvoltage of $\Delta V=3.0 \mathrm{~V}$ and operating temperature of $+23{ }^{\circ} \mathrm{C}$, the time resolution dependency on the deposited energy of the electrons is estimated by varying the level of the discriminator threshold from $10 \mathrm{mV}$ to $80 \mathrm{mV}$ in steps of $10 \mathrm{mV}$.

Figure 12 illustrates the CTR measurements. The optimum time resolution is obtained for a constant fractional threshold of $0.2(20 \%)$ consistent with the previous result of bias voltage scan.

The effects of timing jitter on the CTR are most significant at low discriminator thresholds because at high thresholds, more photo-electrons are created. As the discriminator threshold increases, electrons of higher energy are collected. The deposited energy can be estimated by comparing the average pulse height with the end point of the pulse height spectrum. At a discriminator threshold of $45 \mathrm{mV}$, the average pulse height matches about $1 \mathrm{MeV}$ electrons close to an MIP. At this point, the time resolution is $\sigma_{t}=(42.8 \pm 0.1 \pm 0.5) \mathrm{ps}$. The 
first error is a statistical error from the fit, and the second is a systematic error estimated by considering a temperature uncertainty of $0.1^{\circ} \mathrm{C}$ and a source position uncertainty of $\pm 2 \mathrm{~mm}$.

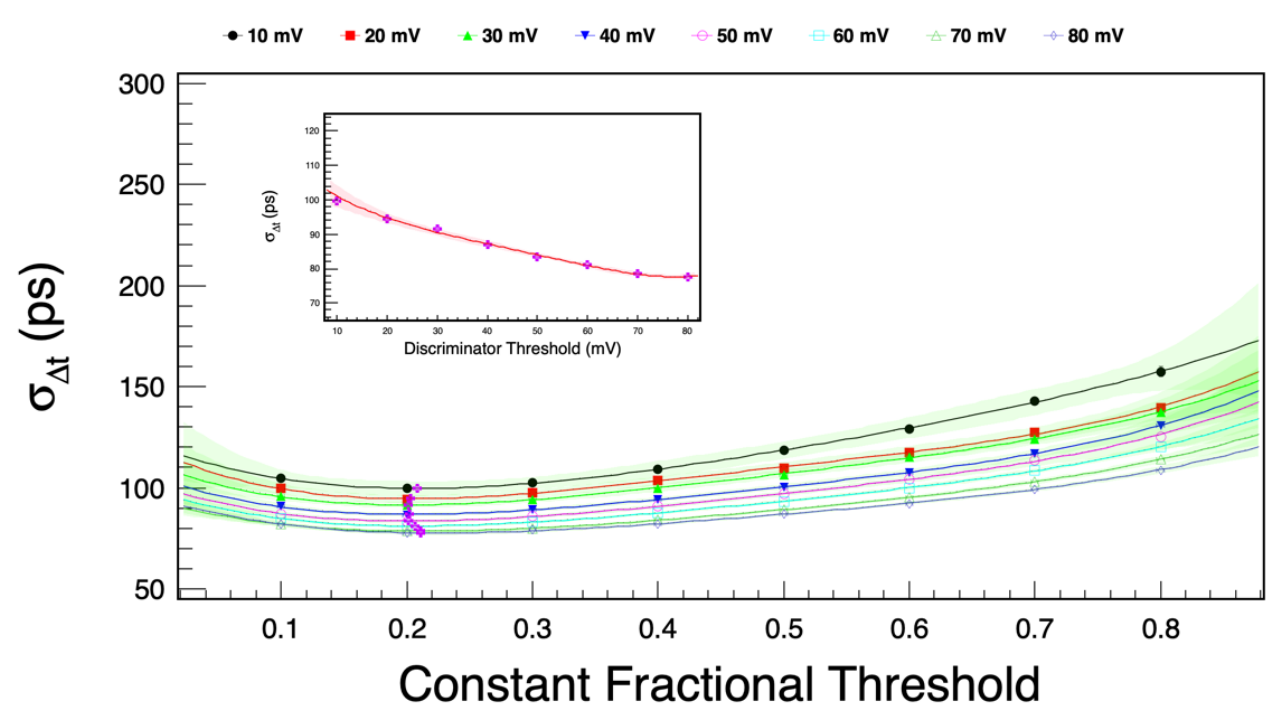

Figure 12. CTR as a function of the constant fractional threshold for different discriminator thresholds that are proportional to the electron energy. The minimum CTR values (magenta points) are distributed around a constant fractional threshold of $0.2(20 \%)$. The inlay shows the minimum CTR as a function of the discriminator threshold. The CTR gradually improves with increasing electron energy from $(98 \pm 0.4)$ ps to $(78 \pm 0.3)$ ps. The colored error bands indicate the $95 \%$ confidence level of the fits.

An external trigger counter was mounted behind the $120 \mathrm{~mm}$ scintillator to select MIPs from ${ }^{90} \mathrm{Sr}$ to validate the time resolution obtained with the internal self trigger. The external trigger counter consists of a $10 \times 10 \times 6 \mathrm{~mm}^{3}$ scintillator (EJ-232) read out by two SiPMs (S14520-6050VS) for a coincidence trigger.

In the external trigger setup, the events are selected by using the same discriminator threshold of $45 \mathrm{mV}$ at $\Delta V=3.0 \mathrm{~V}$. In Figure 13, the pulse heights and the CTR results are compared with the internal trigger mode. In Figure 13a, the external and the internal triggered pulse height spectra are shown. Compared with the internal trigger mode, the mean value of the pulse amplitude is $10 \%$ higher, and the selected events are mostly electrons between 1 and $2 \mathrm{MeV}$, i.e., MIP-like particles [14]. In the internal trigger mode, non-MIP-like low energetic electrons contribute to the signals and worsen the measured time resolution, as shown in Figure 13b. The measured time resolution with the external trigger is $\sigma_{t}=(39.3 \pm 0.1 \pm 0.7)$ ps. The systematic error is estimated by varying the threshold of the external trigger.

\subsection{Time Resolution vs. Operating Temperature}

The sensitivity of the SiPM depends on the product of the photon detection efficiency (PDE) and the gain (G), as described in Equation (1). Both factors depend on the overvoltage $(\Delta V)$, which is strongly correlated with the temperature due to the strong temperature dependency of the breakdown voltage $\left(V_{B D}(T)\right)$, as expressed in Equation (3). This temperature-induced change of $V_{B D}$ affects not only $\Delta V$ but also other characteristics of SiPM, such as PDE, gain, dark count rate, crosstalk and afterpulse probability [19]. However, the light output of the scintillator is constant in the temperature range between $-60^{\circ} \mathrm{C}$ and $+20^{\circ} \mathrm{C}$, as discussed earlier.

The temperature dependency of the CTR is examined by decreasing the operating temperature from $+23{ }^{\circ} \mathrm{C}$ to $-30{ }^{\circ} \mathrm{C}$, while the bias voltage is varied to keep the same overvoltage $(\Delta V)$ according to Equation (4), and the discriminator thresholds are set as 
in Sections 4.5 and 4.6. It should be noted that the bias voltage is applied considering temperature compensation.

To compare the results of the CTR behavior for both temperatures, the CTR is studied as a function of overvoltage and discriminator threshold, as presented in Figure 14.

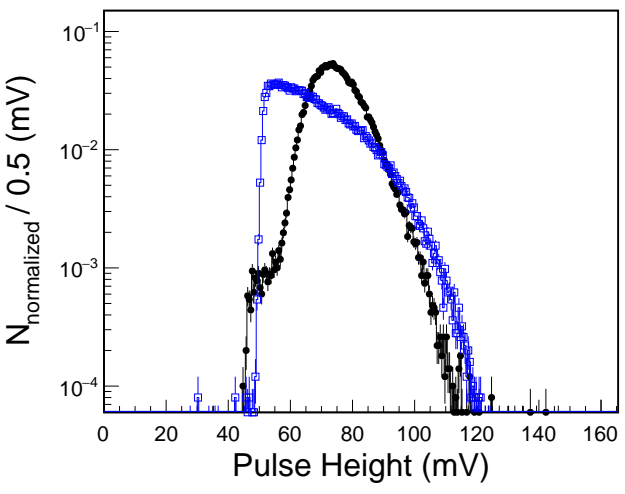

(a)

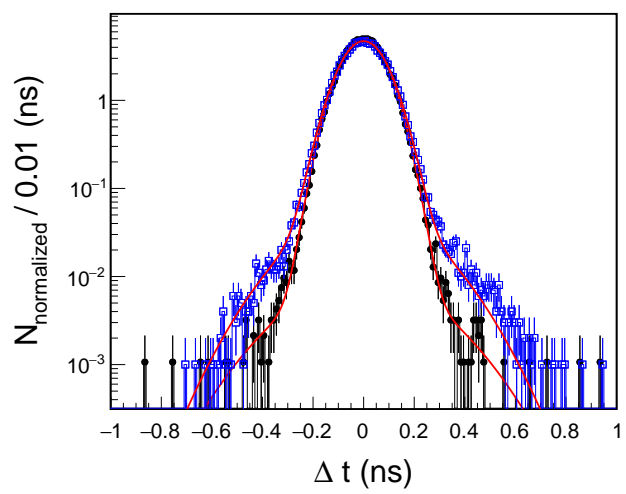

(b)

Figure 13. Comparison of the normalized signal pulse heights (a) and CTRs (b) between external (filled black circle) and internal (open blue square) trigger modes. The mean amplitude of pulse height is $(75.2 \pm 8.6) \mathrm{mV}$ and $(68.7 \pm 12.9) \mathrm{mV}$ for external and internal trigger modes, respectively. The CTR represents the core standard deviation of the Gaussian function and reveals $(78.7 \pm 0.3)$ ps and $(83.3 \pm 0.3)$ ps for external and internal trigger modes, respectively.

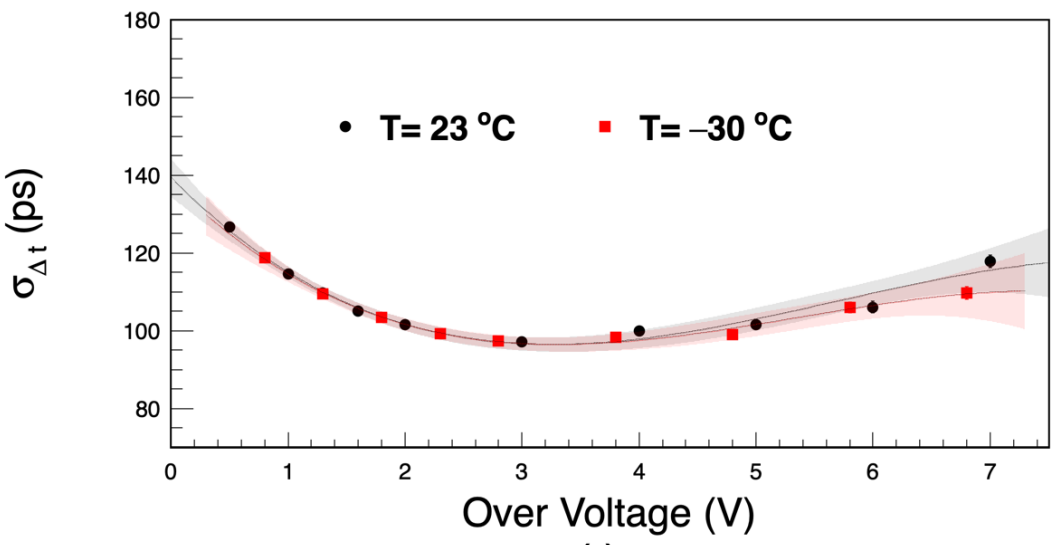

(a)

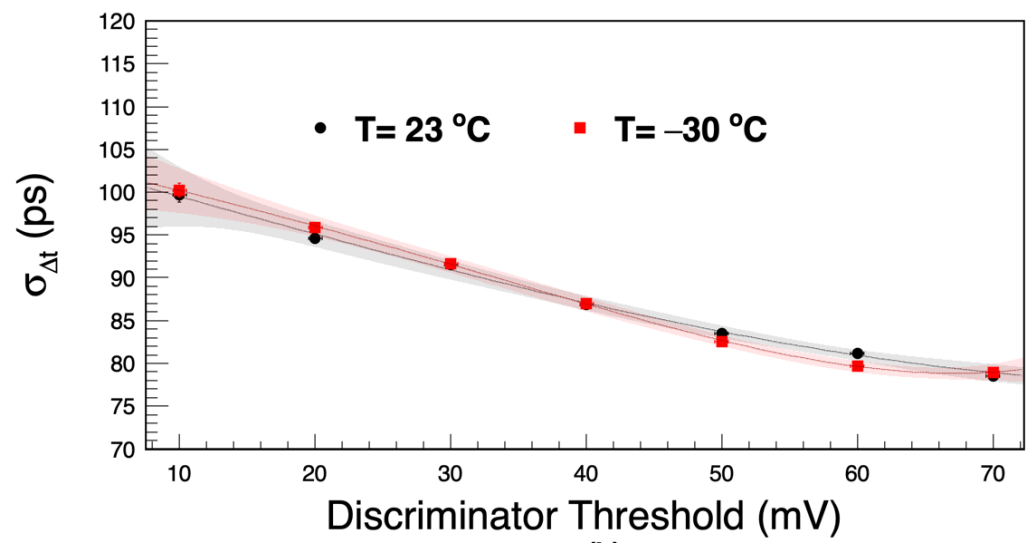

(b)

Figure 14. CTR for two different temperatures as a function of overvoltage (a) and as a function of the discriminator threshold (b). The gray and red error bands indicate the $95 \%$ confidence level of the fits in both cases. 
As already mentioned in Section 3.3, the breakdown voltage $\left(V_{B D}\right)$ shifted from $38 \mathrm{~V}$ to $36 \mathrm{~V}$ as the operating temperature decreased from $+23{ }^{\circ} \mathrm{C}$ to $-30^{\circ} \mathrm{C}$. Figure 14 a shows the measured CTR at a low discriminator threshold of $10 \mathrm{mV}$. For both temperatures, the CTR as a function of overvoltage has a minimum of $\sigma_{t}=(48.2 \pm 0.2)$ ps at $\Delta V=3.25 \pm 0.01 \mathrm{~V}$.

This implies that for the temperature range studied here the time resolution is independent of the temperature, provided that the overvoltage remains the same. A minor difference of the CTR above $\Delta V>5 \mathrm{~V}$ might be the result of the reduced dark counts and the reduced probability of optic crosstalks at cold temperatures [19,20].

In Figure 14b, the CTR is measured as described in Section 4.6 at the same overvoltage of $3.0 \pm 0.1 \mathrm{~V}$ while changing the operating temperature. At $-30{ }^{\circ} \mathrm{C}$, the time resolution reaches $\sigma_{t}=(42.4 \pm 0.5)$ ps at a threshold of $45 \mathrm{mV}$, where the average deposited energy of the electrons is close to an $\operatorname{MIP}\left(E_{e^{-}} \simeq 1 \mathrm{MeV}\right)$. This fact is consistent with the result obtained at $+23{ }^{\circ} \mathrm{C}$.

\section{Conclusions}

A TOF prototype for the AMS-100 experiment has been constructed and its performance has been studied under different operational and environmental conditions. This prototype consists of a $120 \mathrm{~mm}$-long scintillator tile with a surface area of $\left(25 \times 6 \mathrm{~mm}^{2}\right)$ coupled to two SiPMs at its opposite sides. To optimize the time resolution, each SiPM combines four channels into one readout channel by a hybrid connection, and its signal is fed into a high-speed waveform digitizer. By using a radioactive ${ }^{90} \mathrm{Sr}$ source, a time resolution of $\sigma_{t}=[39.3 \pm 0.1$ (stat) \pm 0.7 (syst) $] \mathrm{ps}$ is obtained for MIP-like particles in the external trigger mode.

This result significantly improves the time resolution of $50 \sim 60$ ps reported previously by PANDA and MEG II. This achievement is the result of the use of a new type of SiPM that not only covers the full cross-section of the scintillator at both ends but also offers an improved time resolution over a broad overvoltage range compared to previous SiPMs. Moreover, this work demonstrates the fact that even under different temperature conditions, the time resolution of the TOF counter remains stable provided that the overvoltage is kept the same.

The expected improvement in time resolution of a factor of two from the improved scintillator surface coverage could not be demonstrated. For this purpose, the capacitance (C) of the chosen SiPM (S14161-6050HS, C $=2000 \mathrm{pF}$, area $6 \times 6 \mathrm{~mm}^{2}, 16$ channels) is too large. The SiPM from Hamamatsu (S14161-3050HS, C $=500 \mathrm{pF}$, area $3 \times 3 \mathrm{~mm}^{2}$, 64 channels) will be used to investigate the impact of the capacitance in detail while keeping the scintillator surface coverage at the same level.

In addition, further improvements can be expected from new SiPM technologies [21,22].

Author Contributions: Conceptualization, C.C., T.K. and S.S.; methodology, C.C. and T.K.; software, C.C. and C.D.; validation, T.K. and W.K.; formal analysis, C.C.; investigation, D.L., W.K. and M.W.; resources, T.K., G.S. and S.S.; data curation, C.C., T.B. and C.D.; writing-original draft preparation, C.C.; writing-review and editing, C.C., T.K. and S.S.; visualization, M.W. and C.C.; supervision, S.S.; project administration, G.S. and S.S.; funding acquisition, S.S. All authors have read and agreed to the published version of the manuscript.

Funding: This research was supported by the RWTH Aachen University, Germany.

Institutional Review Board Statement: Not applicable.

Informed Consent Statement: Not applicable.

Data Availability Statement: The data presented in this study are available on request from the corresponding author.

Acknowledgments: We acknowledge the continuous excellent support of the mechanical and electronics workshops of the 1st physics institute, RWTH Aachen University. We thank Sofia Chouridou for the internal review of this article.

Conflicts of Interest: The authors declare no conflict of interest. 


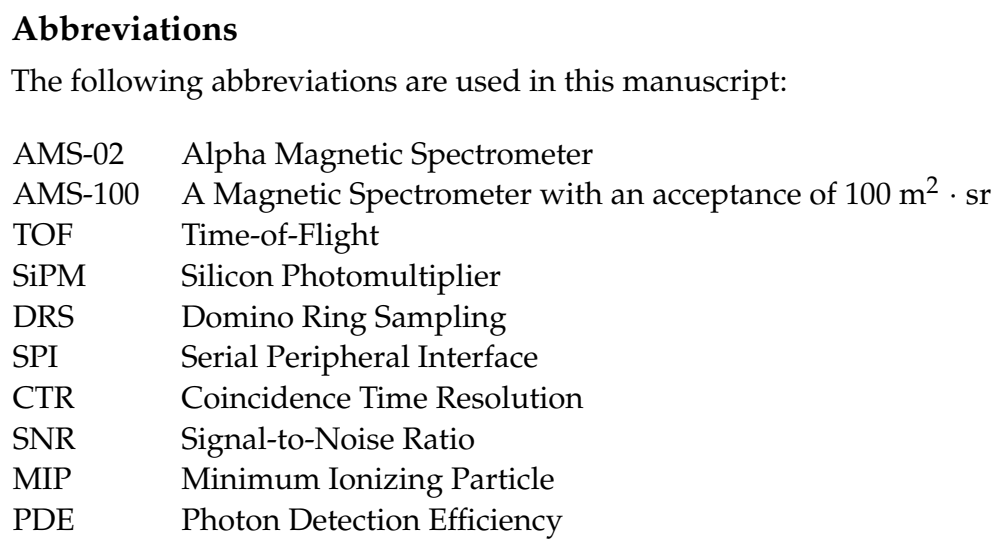

\section{References}

1. Schael, S.; Atanasyan, A.; Berdugo, J.; Bretz, T.; Czupalla, M.; Dachwald, B.; von Doetinchem, P.; Duranti, M.; Gast, H.; Karpinski, W.; et al. AMS-100: The Next Generation Magnetic Spectrometer in Space-An International Science Platform for Physics and Astrophysics at Lagrange Point 2. Nucl. Instrum. Meth. Phys. Res. A 2019, 944, 162561. [CrossRef] [PubMed]

2. Aguilar, M.; Cavasonza, L.A.; Ambrosi, G.; Arruda, L.; Attig, N.; Barao, F.; Barrin, L.; Bartoloni, A.; Başeğmez-du Pree, S.; Bates, J.; et al. The Alpha Magnetic Spectrometer (AMS) on the international space station: Part II-Results from the first seven years. Phys. Rep. 2021, 894, 1-116. [CrossRef]

3. PANDA Collaboration. Technical Design Report for the: PANDA Barrel Time-of-Flight. Available online: https://panda.gsi.de/ system/files/user_uploads/ken.suzuki/RE-TDR-2016-003_0.pdf (accessed on 4 July 2018).

4. Baldini, A.M.; Baracchini, E.; Bemporad, C.; Berg, F.; Biasotti, M.; Boca, G.; Cattaneo, P.W.; Cavoto, G.; Cei, F.; Chiappini, M.; et al The design of the MEG II experiment. Eur. Phys. J. C 2018, 78, 380. [CrossRef]

5. Cattaneo, P.W.; Gerone, M.D.; Gatti, F.; Nishimura, M.; Ootani, W. Development of High Precision Timing Counter Based on Plastic Scintillator with SiPM Readout. IEEE Trans. Nucl. Sci. 2014, 61, 2657-2666. [CrossRef]

6. Vinogradov, S. Approximations of coincidence time resolution models of scintillator detectors with leading edge discriminator. Nucl. Instrum. Meth. Phys. Res. A 2018, 912, 143-153. [CrossRef]

7. Eljen Technology. Available online: https://eljentechnology.com/products/plastic-scintillators/ej-228-ej-230 (accessed on 28 June 2019).

8. Renker, D.; Lorenz, E. Advances in solid state photon detectors. J. Instrum. 2009, 4, P04004. [CrossRef]

9. Yamamoto, K.; Nagano, T.; Yamamda, R.; Ito, T.; Ohashi, Y. Recent Development of MPPC at Hamamatsu for Photon Counting Applications. In Proceedings of the 5th International Workshop on New Photon-Detectors (PD18), Tokyo, Japan, 27-29 November 2018.

10. Böhm, M.; Lehmann, A.; Motz, S.; Uhlig, F. Fast SiPM Readout of the PANDA TOF Detector. J. Instrum. 2016, 11, C05018. [CrossRef]

11. DRS4 Evaluation Board. Available online: https://www.psi.ch/en/drs/evaluation-board (accessed on 3 June 2020).

12. Datasheet of the Hamamatsu C11204-01. Available online: https://www.hamamatsu.com/resources/pdf/ssd/c11204-01_kacc1 203e.pdf (accessed on 9 February 2021).

13. Datasheet of the FT4222H Chip. Available online: https:/ ftdichip.com/products/ft4222h (accessed on 28 October 2020).

14. NIST-ESTAR Program. Available online: https://physics.nist.gov/PhysRefData/Star/Text/ESTAR.html (accessed on 2 December 2020).

15. Gedcke, D.A.; McDonald, W.J. A constant fraction of pulse height trigger for optimum time resolution. Nucl. Instrum. Meth. 1967, 55, 377. [CrossRef]

16. Gedcke, D.A.; McDonald, W.J. Design of the constant fraction of pulse height trigger for optimum time resolution. Nucl. Instrum. Meth. 1968, 58, 253. [CrossRef]

17. Seifert, S.; Van Dam, H.T.; Vinke, R.; Dendooven, P.; Lohner, H.; Beekman, F.J.; Schaart, D.R. A Comprehensive Model to Predict the Timing Resolution of SiPM-Based Scintillation Detectors: Theory and Experimental Validation. IEEE Trans. Nucl. Sci. 2012, 59, 190. [CrossRef]

18. Acerbi, F.; Gundacker, S. Understanding and simulating SiPMs. Nucl. Instrum. Meth. A 2019, 626, 16-35. [CrossRef]

19. Nepomuk Otte, A.; Garcia, D.; Nguen, T.; Purushotham, D. Characterization of Three High Efficiency and Blue Sensitive Silicon Photomultipliers. Nucl. Instrum. Meth. A 2017, 846, 106. [CrossRef]

20. Collazuol, G.; Bisogni, M.; Marcatili, S.; Piemonte, C.; Guerra, A.G. Studies of silicon photomultipliers at cryogenic temperatures. Nucl. Instrum. Meth. A 2011, 628, 389-392. [CrossRef]

21. Lecoq, P. Pushing the Limits in Time-of-Flight PET Imaging. IEEE Trans. Radiat. Plasma Med. Sci. 2019, 1, 473-485. [CrossRef]

22. The 10 ps TOF-PET Challenge. Available online: https://the10ps-challenge.org (accessed on 11 September 2021). 